\title{
Recovery of intralipid from lumbar puncture after migration of saphenous vein catheter
}

\author{
Felix Odaibo, Carlos A Fajardo, Catherine Cronin
}

\begin{abstract}
A term female infant was admitted to the intensive care unit with the diagnosis of tetralogy of Fallot with critical pulmonary stenosis. On the seventh day of life a long saphenous line was inserted that remained without complications until seven days later when the infant appeared septic. A lumbar puncture demonstrated the presence of intralipid in the cerebrospinal fluid that we interpreted as due to migration of the saphenous catheter. The child had an uneventful recovery.
\end{abstract}

\section{(Arch Dis Child 1992;67:1201-3)}

Newborns requiring long term parenteral nutrition have been managed with central venous catheters. Between 28-50\% of these infants develop complications of catheterisation. ${ }^{12}$ Complications are associated with low birth weight, especially less than $1000 \mathrm{~g}$, lower gestational age, and a longer duration of catheter insertion as well as repeated catheterisation. ${ }^{1}$ In this report, we describe a term baby who developed late extravasation of a long saphenous vein line into the epidural space.

\section{Case report}

An infant girl delivered at term by caesarean section weighed $3260 \mathrm{~g}$ and required no active resuscitation. Apgar scores were 9 and 9 at one and five minutes respectively. The child was transferred from another hospital to this centre for investigation of intermittent circumoral cyanosis. Investigations confirmed tetralogy of Fallot with critical pulmonary stenosis. She was managed with prostaglandin infusion. Balloon angioplasty was carried out on the fourth day of life through the right saphenous vein after failure to get the catheter through the umbilical vein. The procedure was complicated by difficulty in passing the catheter through the saphenous vein.

On the seventh day of life, the baby developed necrotising enterocolitis necessitating the institution of total parenteral nutrition. A long line (Dow Corning Silastic Medical) was established with ease on the eighth day of life via the left saphenous vein. Confirmation of its position in the femoral vein was carried out by withdrawing blood from the catheter and an $x$ ray film (fig 1).

On the 15th day of life, seven days after insertion of the central venous catheter, she became febrile and mottled, with poor peripheral perfusion. A blood count showed neutrophilia with a leucocyte count of $51 \times 10^{9} / 1$ and young cells of $30 \%$. A lumbar puncture revealed a milky fluid that was not under pressure and contained a triglyceride concentration of 42.6 $\mathrm{mmol} / \mathrm{l}$. No cells were found and there was no growth on culture. Fluoroscopy showed opacification of the epidural space with the tip of the catheter at lumbar level 4 and 5 (fig 2). Blood could not be withdrawn from the catheter so it was promptly removed. An electroencephalogram, ultrasound scan, and computed tomogram of the spine were normal with no evidence of masses within the epidural space. Repeat lumbar puncture one week after the incident was completely normal.

A magnetic resonance imaging scan of the spine carried out about the seventh week of life revealed only questionable narrowing of L4-L5 disc space with no evidence of cord compression or expansion and neither extradural abnormality nor aberrant vessels were identified. The child made an uneventful recovery but was readmitted with small bowel obstruction due to segmental volvulus. Postoperatively she had no complications.

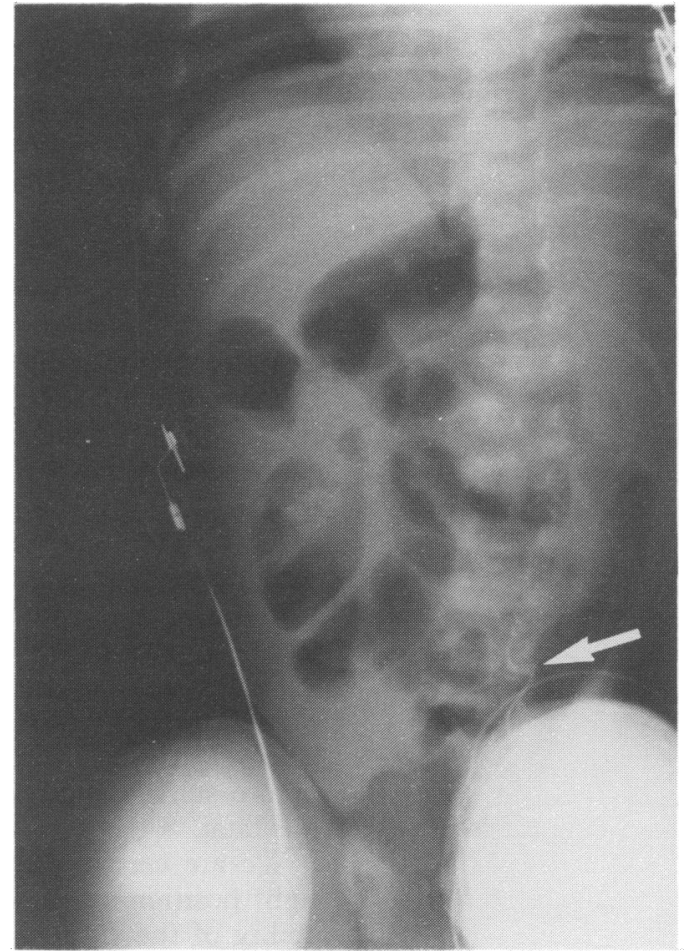

Figure 1 Radiograph contrast study immediately after surgical insertion of Silastic catheter in the left femoral vein. Note the arrow pointing to the tip of the catheter.
Canada R3E 0L.8.

Accepted 10 April 1992 


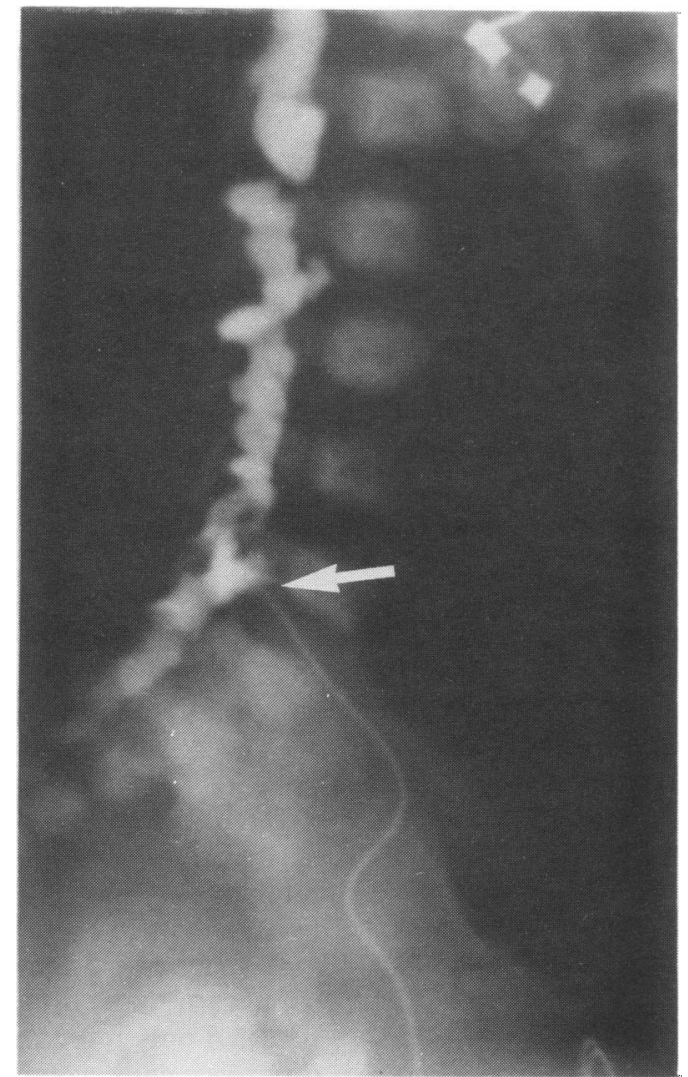

Figure 2 Lateral radiograph during fluoroscopy. Note that the contrast material outlines the epidural space and the arrow points to the catheter traversing the L4-L5 disc space.

\section{Discussion}

Complications of catheter placement are widely reported. The most common complications are thrombosis and infection. The causal organisms include Staphylococcus epidermidis, Staphylococcus aureus, Pseudomonas aeruginosa, and Candida albicans. ${ }^{2}$ Other complications include catheter rupture, dislodgement, failure, leakage of fluid, and peripheral oedema.

The more serious complications relate to cardiorespiratory compromise. Respiratory difficulty may occur as a result of collections in the pleural cavity. ${ }^{3}$ Complications are no more frequent with indwelling femoral central lines than with lines in other sites. ${ }^{4}$ This report demonstrates that even with careful checking of the position of the catheter, displacement or migration of the catheter can still occur. A high index of suspicion is required to alert a physician to this rare type of complication. In another case reported catheterisation of the femoral vein via the left saphenous vein resulted in the infusion of the contents of parenteral nutrition into the dural and retroperitoneal spaces. ${ }^{5}$ Whereas the clinical presentation and lumbar puncture findings were similar to ours, the position of their catheter was never established before starting the infusion, and the outcome was fatal.

We are certain that the catheter was in the right position when first placed because of the ability of the surgeon to withdraw blood from the line soon after its insertion and the radiological confirmation of its position. It must have been in place until the very end as this infant received parenteral nutrition over a seven day period through this line at a rate of $150 \mathrm{ml} / \mathrm{kg} /$ day and had a sudden deterioration with no recovery of blood from the line at that time.

One can only speculate as to the mechanisms of this rare complication. The veins of the vertebral canal form freely communicating plexuses along its length both inside and outside the canal. The internal vertebral plexuses form a continuous network between the dura mater and the walls of the vertebral canal. Because these vessels are so thin walled, it is probable that the catheter, having passed into the ascending lumbar veins, entered the intervertebral veins, ruptured its wall, thereby lodging in the epidural space (figs 3 and 4). We postulate that obstruction or thrombosis of femoral vessels may lead to increased blood flow through the paravertebral plexus, which may displace the tip of the catheter from its original position. However, we were not able to confirm this on radiological examination.

Our case report emphasises that long Silastic central catheters may become displaced at any time, even when correct placement has been documented radiologically. Furthermore, there may be no external evidence that displacement has taken place. We recommend (i) that manufacturers imprint catheters with indelible marks so that the length of tubing inside the body may be documented, (ii) that the catheters tip be radiopaque, enabling displacement to be identified on a plain $x$ ray film, and (iii) that personnel caring for infants with central venous Silastic

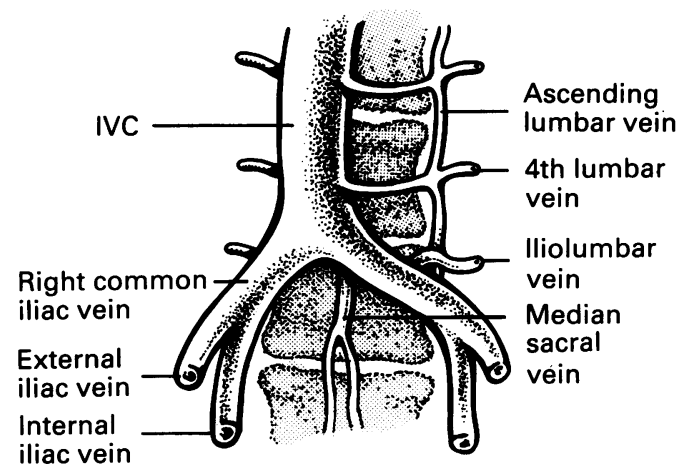

Figure 3 Inferior vena cava (IVC) and its tributaries.

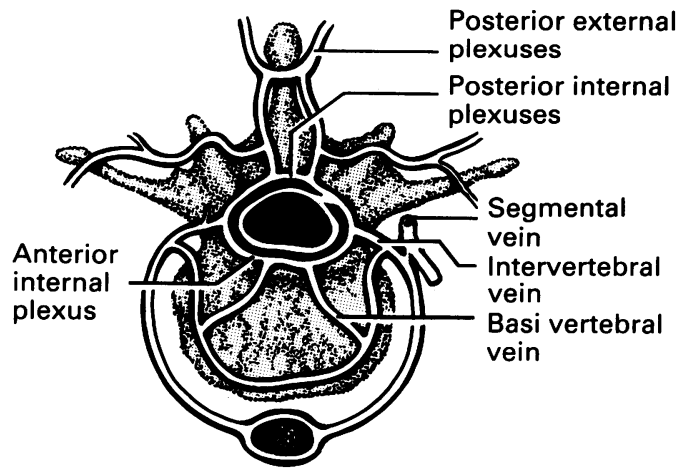

Figure 4 Venous supply of lumbar vertebrae. 
catheters have a high index of suspicion of this complication, which may be asymptomatic.

1 Grisoni ER, Mehta SK, Connors AF. Thrombosis and infection complicating central venous catheterization in neonates. $\mathcal{f}$ Pediatr Surg 1986;21:772-6.

2 Richet $H$, Hubert B, Nitemberg G, et al. Prospective multicenter study of vascular-catheter-related complications and risk factors for positive central-catheter cultures in intensive care unit patients. $\mathcal{J}$ Clin Microbiol 1990;28:2520-5.

3 Rubin S, Hewson P, Roberton NRC. Pulmonary complications of total parenteral nutrition in a neonate. $\mathcal{F} R$ Soc Med 1986;79:545-7.

4 Kanter RK, Zimmerman JJ, Strauss RH, Stoeckel KA. Central venous catheter insertion by femoral vein: safety and effectiveness for the pediatric patient. Pediatrics 1986; 77:842-7.

5 Kelly MA, Finer NN, Dunbar LG. Fatal neurologic complication of parenteral feeding through a central vein catheter. Am $\mathcal{F}$ Dis Child 1984;138:352-3. 